\title{
LabVIEW based Abnormal Muscular Movement and Fall Detection using MEMS Accelerometer during the Occurrence of Seizure
}

\author{
P. Grace Kanmani Prince ${ }^{1}$, Rani Hemamalini ${ }^{2}$ and R. Immanuel Rajkumar ${ }^{1}$ \\ 'Sathyabama University, Chennai, India; coggrace05@gmail.com \\ ${ }^{2}$ St. Peter's College of engineering and Technology, Chennai, India
}

\begin{abstract}
The manifestation of seizure in patients can be in the form of abnormal muscular movement occurring at the extremities and it can cause sudden fall to the patients which can lead to injuries. MEMS accelerometer MPU6050 is used to acquire the signals from the extremities to detect abnormal movements and sudden fall. The signals are received through an interfacing device named Arduino Uno and are processed using LabVIEW software. The Wavelet transform is used for detecting the changes in movement of the extremity and a thresholding technique is used for seizure detection and fall detection. Once a seizure or fall is detected then a message is sent to the caretaker through GSM.
\end{abstract}

Keywords: Abnormal Muscular Movement, Fall Detection, Mems Accelerometer, Seizure, Wavelet Transform

\section{Introduction}

A seizure occurs when a burst of electrical impulses in a part of the brain crosses its normal limit. It spreads to neighboring areas and all other parts of body which creates an uncontrolled storm of electrical activity. These electrical impulses are transmitted to the muscles, causing twitches or convulsions. The signs and symptoms of seizures vary differently for each patient. It may cause involuntary changes in body movement, function, sensation, awareness, or behavior. Seizures are most often associated with a sudden and involuntary muscular contraction at the extremities and loss of consciousness for a certain period of time.

The most common tools used for seizure detection are EEG and video recording. But wearing the EEG electrodes and being confined to a place for video recording for a longer duration can be of great discomfort and limits the mobility of the patient. Hence other body signals such as muscular movement, heart signals, and respiration rate and humidity sensor are now used to detect the seizure activity $^{1}$. Since most of the seizures are accompanied by muscular convulsions an effective method for automatic detection of seizures is observing the muscular activity during the time of seizure. Hence for detecting the muscular movement at the extremities accelerometer proves to be the best sensor which is able to detect the contraction of muscles during the time of seizure. MEMS accelerometers are very compact and can be easily worn at the extremities as wrist watches ${ }^{2}$ or wrist bands ${ }^{3}$.

In this paper the muscular activity is detected with the help of MEMS sensor which is attached to the hand of the subject. The signals received from the sensor are used to classify the normal and abnormal activity. The MEMS sensor used here is MPU6050 which contains a three axis accelerometers and Gyroscope. The MPU6050 is interfaced with the computer through Arduino Uno. The accelerometer data is received in LabVIEW for further

*Author for correspondence 
processing and classification. The advantages of this module include high speed, low power and cost effective components.

The Section 2 of this paper gives the Hardware details; Section 3 gives the details of the algorithms used to detect activity, fall detection and abnormal muscular movement during the occurrence of seizure. Section 4 gives the results and Section 5 provides the concluding remarks.

\section{Hardware Descriptions}

The hardware setup consists of MEMS sensor MPU6050 to detect the muscular movement at the extremity. This sensor is interfaced with the computer through Arduino UNO.

\subsection{MPU6050}

The MPU6050 is a compact motion processing technology. It consists of a 3-axi+s gyroscope and a 3-axis accelerometer. The three axes are present to acquire the magnitude and direction of acceleration and orientation. Accelerometer and Gyroscope are combined on a single silicon chip with an onboard digital processor. It is capable of tracking fast and slow motion. The main advantages of MPU6050 are low cost and low power (3.3V). It is a high performance miniature device. It is an I2C devise which can communicate at a maximum speed of $400 \mathrm{kHz}$.

\subsection{Arduino Uno}

The Arduino Uno is an open platform circuit which is easy to access. It is an ATmega32 based microcontroller board. There are 14 pins available for digital inputs and outputs. Another 6 pins are available for analog operations. It consists of $16 \mathrm{MHz}$ clock which enables it to work fast. The board is connected to PC through USB cable. It is powered by external supply or through USB connection. It consists of a $32 \mathrm{~KB}$ flash memory to store the code. MPU6050 is connected to the analog pins of Arduino board.

\section{Algorithm for Seizure and Fall Detection}

A frame work having a binary decision tree was proposed by M. J. Mathie et $\mathrm{al}^{4}$. Based on this frame work an algorithm for automatic detection of seizure and fall is explained in the flow chart given in Figure 1. The MEMS

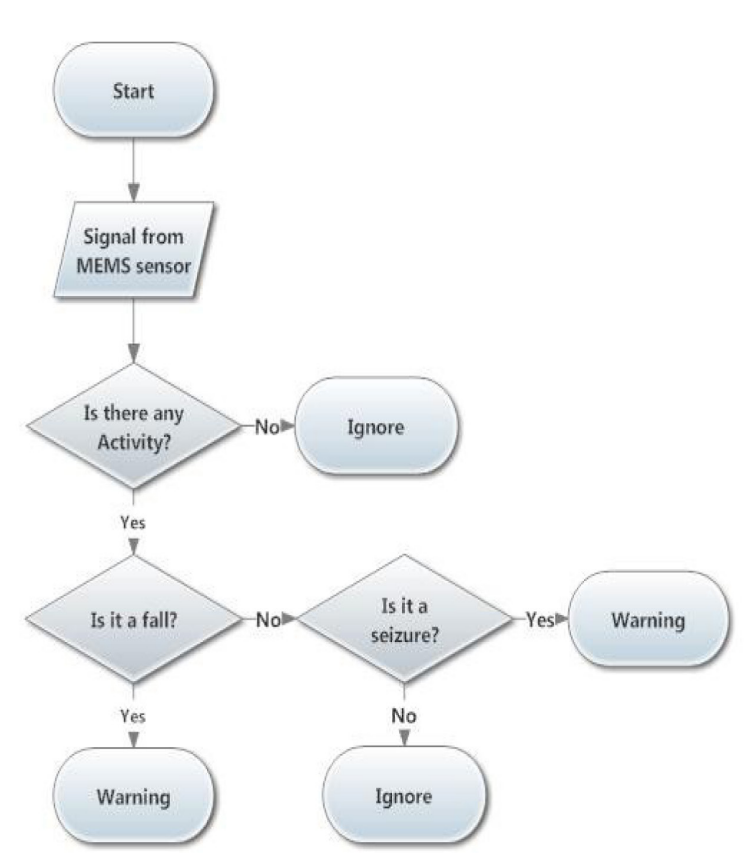

Figure 1. Flow Chart of the Seizure and Fall detection Algorithm.

sensor is placed at the extremity preferably in the hand. Through the MEMS sensor signals are acquired and analyzed. If there is an activity the amplitude and frequency of the signals from the MEMS sensor changes depending on the nature of activity. If the activity is detected as fall then warning is given or else the signal is analyzed whether it is a normal activity or a seizure activity. If it corresponds to a seizure activity then warning is given.

\section{Methodology}

\subsection{Data Acquisition}

MPU6050 is a MEMS sensor that contains three axis accelerometer and three axis gyroscope. Three axis Accelerometer data is available and it is acquired through Arduino Uno and read through serial port into LabVIEW. The data that is available is in the form of a string variable. It is then converted into a numerical variable. The three axis data are acquired separately and their vector magnitude is found by calculating the root mean square of the data available at all the three axes.

$$
V_{r m s}=\sqrt{a_{x^{2}}+a_{y^{2}}+a_{z^{2}}}
$$

The data acquisition is created as a Sub VI which has its input from the data read from the serial port. Its output 
consists of numerical values of the analog voltages that are proportional to the acceleration that is parallel to each axis. The vector magnitude is also available as a fourth output. The function blocks used here are mostly point by point VIs.

\subsection{Activity Detection}

Przemys ławs Korohoda et al have performed detail study of seizure activity using Short time Fourier transform ${ }^{5}$. When STFT was used the detection of activity took much longer time duration. Hence wavelet transform was used for detection of activity. The activity is detected by obtaining point by point wavelet transform of the vector magnitude. The results obtained by applying Wavelet transform gave instantaneous response. The mean of the wavelet transform is calculated. The rate of change of mean is obtained. A threshold is fixed for the differential value of mean. If the differential value exceeds the threshold limit then the activity is detected. The sensor is very sensitive to minute changes and could possibly show difference in values of the output for insignificant changes. Hence a threshold value is fixed to detect the actual movement. If the rate of change of mean increases above the threshold it is taken as activity or else it is taken as rest. The LabVIEW block diagram for activity detection is given in Figure 2 and the results are shown in Figure 3 for rest state where the differential value is below the threshold and Figure 4 shows the state of activity above the differential value is above the threshold.

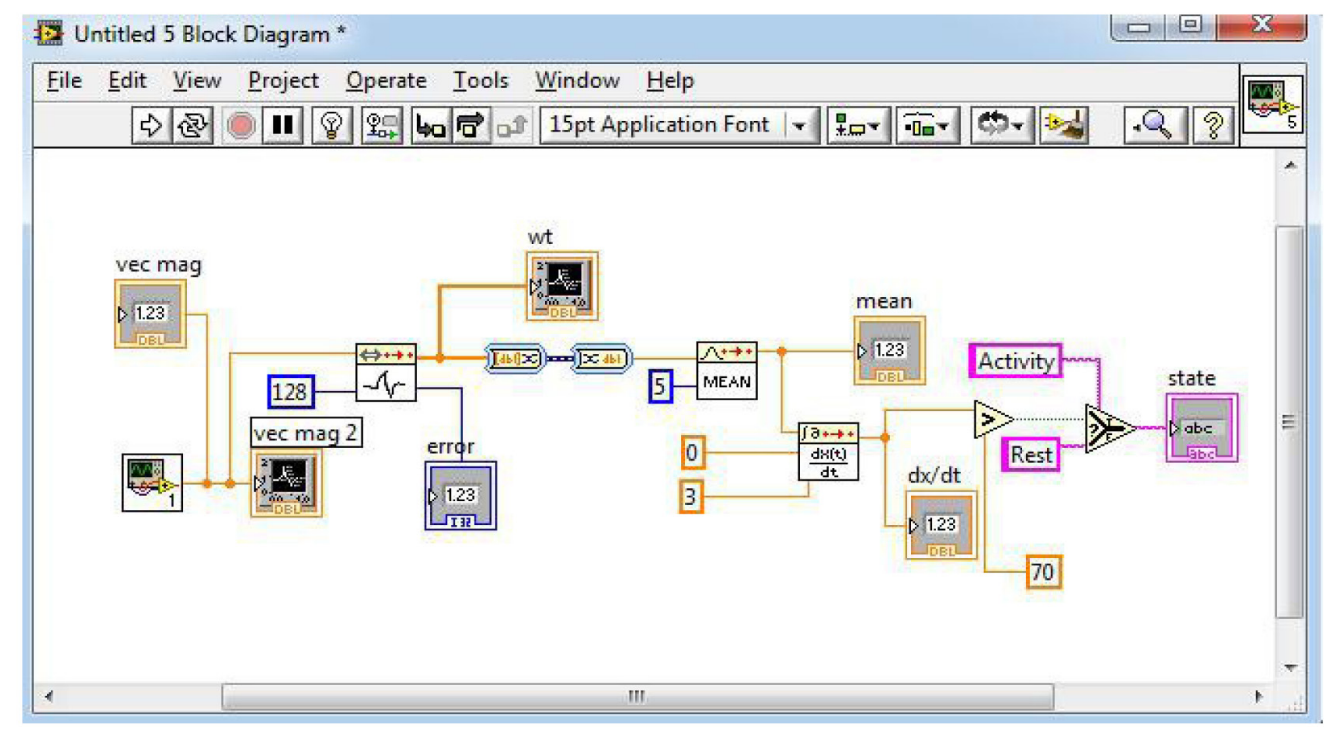

Figure 2. Rest and Activity detection.

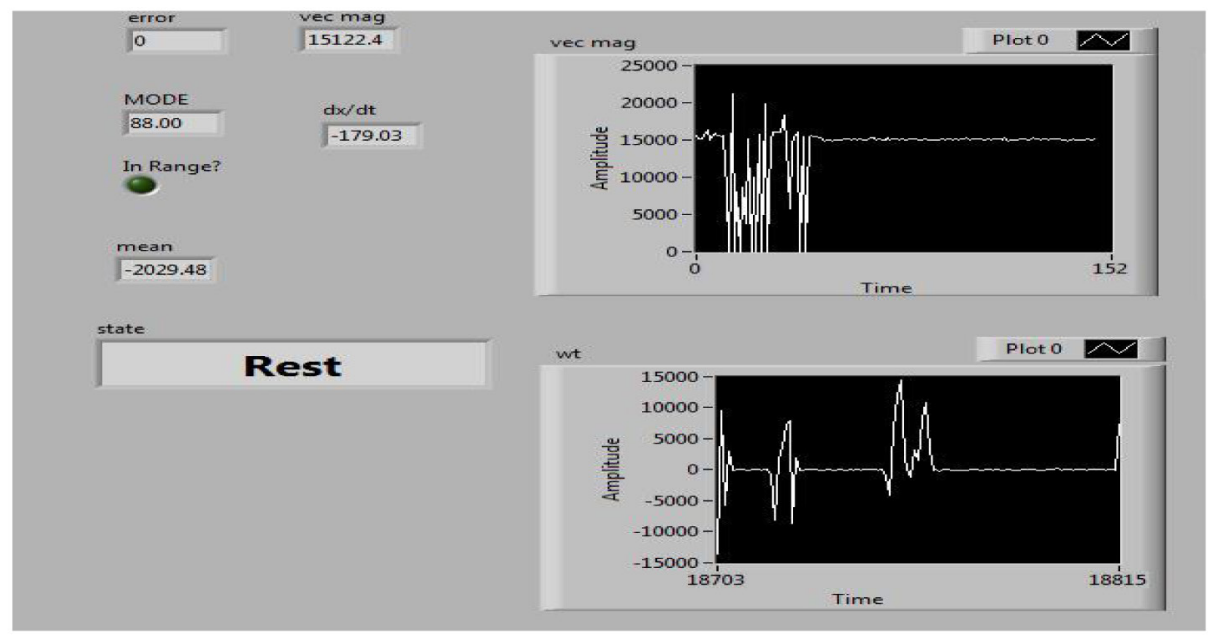

Figure 3. Output observed in rest state. 


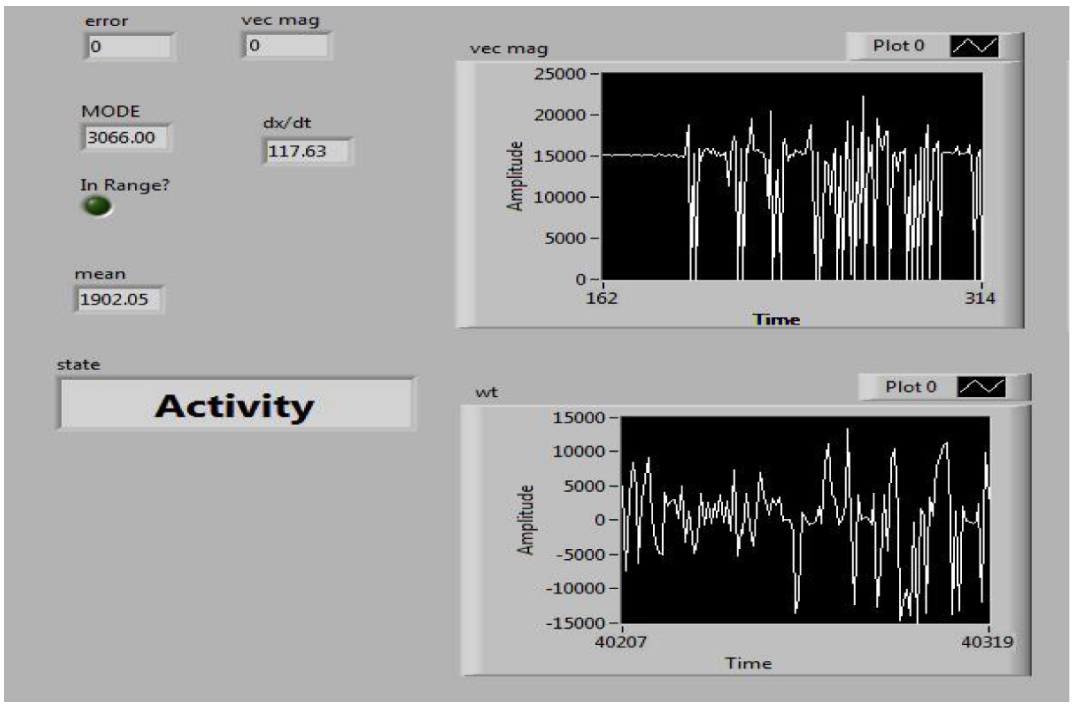

Figure 4. Output observed in activity state.

\subsection{Fall Detection}

For fall detection the sum of the actual values from the accelerometer are taken. The values of the accelerometer increases as the sensor moves towards ground since the values are influenced by the gravitational force. Certain values of consecutive samples are queued up. For example say 30 samples are queued. The difference between the current sample and a certain past sample is calculated. If the difference is above a threshold value then it is considered as fall. The difference is considered instead of the actual reading because the accelerometer value varies with respect to the position of the sensor from the ground. For example the values obtained in the ground floor of the building will be very much different from the values obtained at the first floor. The threshold has to be fixed since a false alarm can be triggered when a person shifts from standing to sitting position. Hence the difference in the signal has to be noted appropriately as given by Dean M. Karantonis et $\mathrm{al}^{6}$. Ones the fall is detected the signal is given to the zero order hold block which hold the samples for the given number of samples. Since the difference is considered instead of the actual values, even when the person remains in fall state the difference becomes lesser in few seconds. The zero order hold holds the input data points for the given number of cycles. Figure 5 shows the LabVIEW block diagram of fall detection and Figure 6 shows the output of normal condition when the difference is lesser than the threshold value. Figure 7 shows output at fall state when the difference exceeds the threshold value.

\subsection{Seizure Detection}

The seizure activity is manifested externally through the extremities in the form of periodic convolutions. The frequency and amplitude available from the signals of the MEMS sensor will be constant at the time of seizure. The patterns of muscular movement for different types of seizure are explained by Tamara M. E. Nijsen et $\mathrm{al}^{7}$. The amplitude and frequency will be lesser when compared to rigorous activities like walking, running etc. It would be higher when compared to other moderate activities like eating. Hence histogram of certain number of consecutive data points is obtained. The largest bin in the histogram is found and the midpoint of the largest bin is obtained which gives the mode of the vector magnitude. If the mode value falls into a particular range for a given period of time then it is expected to be the occurrence of seizure. Hence if the mode falls into the prescribed range and remains in that range for the given time then seizure warning is given ${ }^{8}$. Certain activities like scratching and erasing also can give similar results hence a time limit is given. If the time exceeds the limit then seizure warning is given. The results are given in Figure 8 and Figure 9. This method can also be used with EEG, video recording and ECG sensors for monitoring the seizure patients and it will enhance the detection of the occurrence of seizure more effectively ${ }^{9}$.

Once an abnormality is detected, immediately a message is sent to the mobile phone of the caretaker through GSM module. The Figure 10 shows the data read from the accelerometer and the sending of message when a fall 


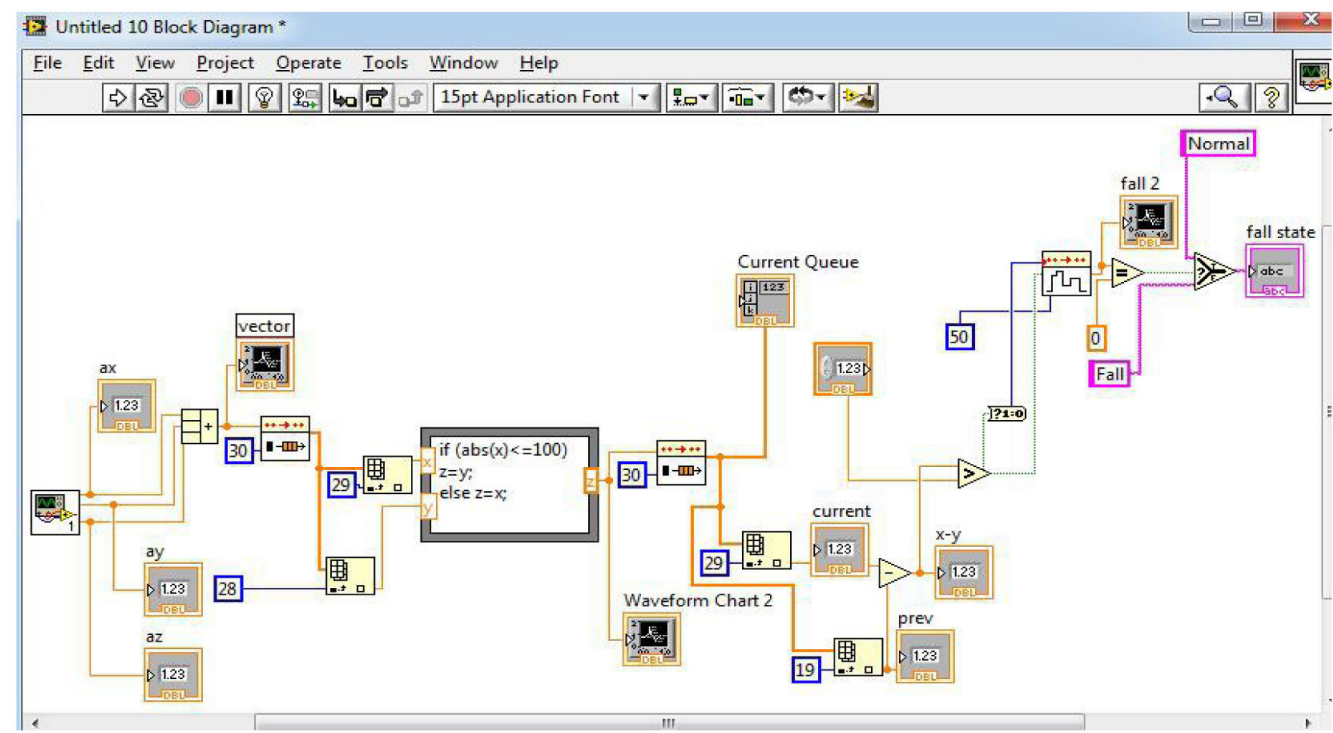

Figure 5. Block diagram for fall detection.
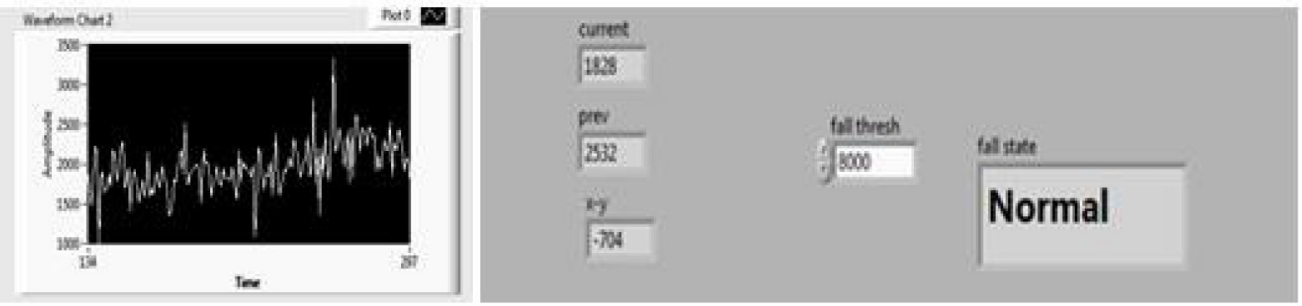

Figure 6. Output displaying Normal condition.
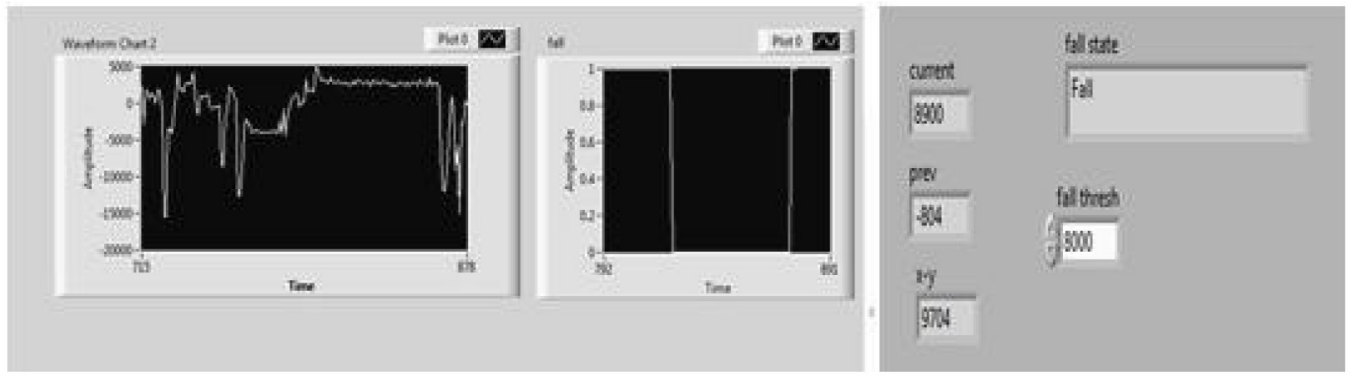

Figure 7. Output displaying fall condition.

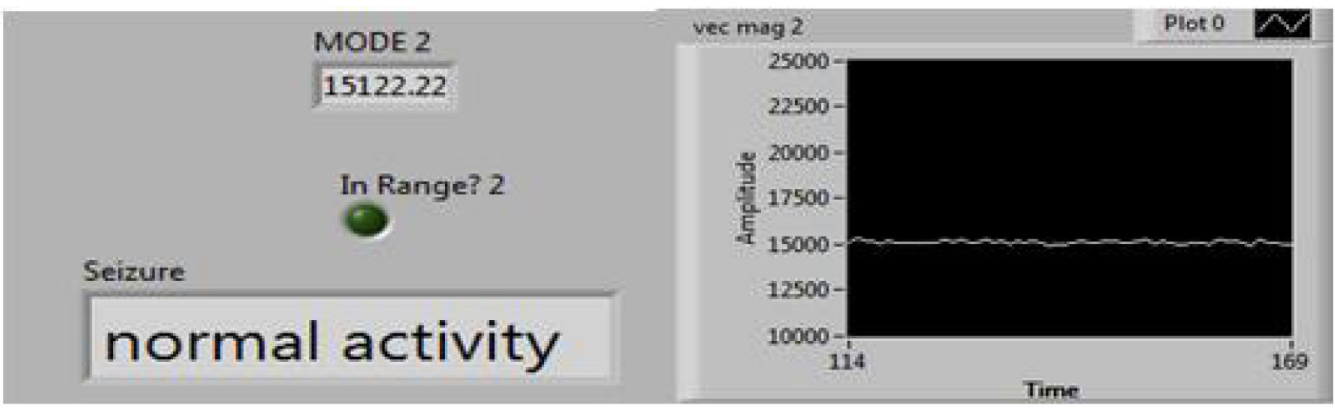

Figure 8. Normal Activity. 


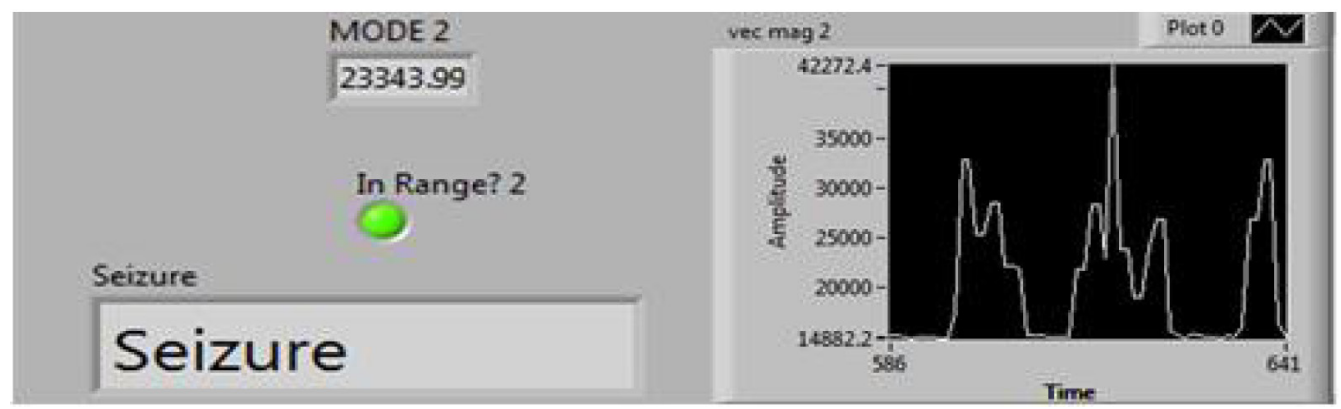

Figure 9. Seizure activity.

\begin{tabular}{|c|c|c|c|c|c|}
\hline 50 & 19 & 110 & 147 & 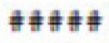 & NORMAL \\
\hline 72 & 50 & 19 & 110 & 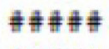 & NORMAL \\
\hline 478 & 72 & 50 & 19 & 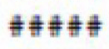 & SEND SMS \\
\hline 582 & 478 & 72 & 50 & 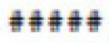 & FALL \\
\hline 1 & 582 & 478 & 72 & $\# \# \# \#$ & FALL \\
\hline 5 & 1 & 582 & 478 & 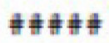 & FALL \\
\hline
\end{tabular}

Figure 10. Accelerometer data and sending of SMS.

is detected. GSM module is helpful for transmitting the patient's condition and can be useful in ensuring patients safety and quick attention can be given to the patient ${ }^{10}$.

\section{Conclusion}

MEMS sensor MPU6050 proves to be an effective tool for detection of seizure. It is more compact and easy to fix in the extremities. The cost of the components used is also economical. It can be made wireless and designed in the form of wrist watches. Wavelet transform is an effective time frequency domain analysis tool which gives a faster response when compared to STFT. Thresholding is the major concept used in this work. Activity detection, fall detection and seizure detection are all based on Thresholding technique. In activity detection the value of the wavelet coefficient is considered. If the wavelet coefficient exceeds a threshold limit it is considered as activity. Fall is detected by fixing a threshold of the difference between the present and past sample. Seizure is detected by calculating the mode of the frequency of the signal that is obtained from the sensor. If the mode value falls within in a certain range and remains in the range for certain amount of time then it is considered to be a seizure. When fall or seizure is detected a message is passed to the care taker through GSM.

\section{References}

1. Vel AVD et al. Non-EEG seizure-detection systems and potential SUDEP prevention: State of the art, Seizure. European Journal of Epilepsy. 2013 Jul; 22(5):345-55.

2. Borujeny GT, Yazdi M, Haddad AK, Borujeny AR. Detection of Epileptic Seizure Using Wireless Sensor Networks. Journal of Medical Signals and Sensors. 2013.

3. Ming-Zher P. Continuous Assessment of Epileptic Seizures with Wrist-worn Biosensors. [PhD Thesis]. Massachusetts Institute of Technology; 2011.

4. Mathie MJ, Celler BG, Lovell NH, Coster ACF. Classification of basic daily movements using a triaxial accelerometer. Med Biol Eng Comput. 2004; 42:679-87.

5. Korohoda PL, Kołodziej J, Stępień J. Time-frequency analysis of accelerometry data for seizure detection. BioAlgorithms and Med-Systems. 2013; 9(2):65-71.

6. Karantonis DM, Narayanan MR, Mathie M, Lovell NH, Celler BG. Implementation of a real-time human movement classifier using a triaxial accelerometer for ambulatory monitoring. IEEE Transactions on Information Technology in Biomedicine. 2006; 10: 156-67. 
7. Nijsen TME, Aarts RM, Arends JBAM, Cluitmans PJM. Automated detection of tonic seizures using 3-D accelerometry. IFMBE Proceedings ECIFMBE. 2008; 22:188-91.

8. Burchfield RT, Venkatesan S. Accelerometer-based human abnormal movementdetection in wireless sensor networks. HealthNet '07 Proceedings of the 1st ACM SIGMOBILE International Workshop on Systems and Networking Support for Healthcare and Assisted Living Environments. 2011; 67-9.
9. Nijsen TME. Accelerometry based detection of epileptic seizures. [PhD Thesis]. Technische Universiteit Eindhoven; 2008. ISBN- 978-90-386-1379-6.

10. Singh RR. Preventing Road Accidents with Wearable Biosensors and Innovative Architectural Design. 2nd ISSS National Conference on MEMS, Microsensors, Smart Materials, Structures and Systems; 2007. 